British Journal of Psychiatry (1995), 166, 262-268

\title{
Correspondence
}

Contents: Asperger's syndrome and violence/ Mental health reforms in Europe/ECT seizure threshold and fluoxetine/Discrepancies on prescribing antipsychotics/Lithium in steroid-induced depression/Language and psychiatry/Psychiatrists and priests/Comparing treatments for generalised anxiety disorder.

\section{Asperger's syndrome and violence}

SIR: Scragg \& Shah's paper on the prevalence of Asperger's syndrome (AS) in Broadmoor Hospital (BJP, November 1994, 165, 679-682) is important because it alerts us to a group of people in special hospitals whose pattern of disability means that they have special needs and may be particularly vulnerable. Indeed there may be others with undiagnosed AS elsewhere in the criminal justice system.

Scragg \& Shah assert that the prevalence of AS is significantly greater in Broadmoor Hospital than in the general community and that this supports an association between AS and violent behaviour. We would dispute whether the Ehlers \& Gillberg (1993) study took a representative sample of the general community with which the Broadmoor population can be compared. They studied people from a different country (Sweden), of a different age (schoolchildren), of a different social background (predominantly middle class) and different educational background (all attended mainstream school). We therefore do not think that any difference in prevalence between the two groups can necessarily be attributed to an increased tendency for violent behaviour in the Broadmoor sample.

We would also dispute whether a significant difference in prevalence has in fact been demonstrated. Ehlers \& Gillberg do not unfortunately report confidence intervals for their male subsample, but even taking their (lower) figures for the whole sample, we find that the confidence intervals in the two studies overlap. For definite AS, Ehlers \& Gillberg (1993) found a prevalence of $0.36 \%(95 \%$ CI $0.11 \%-0.84 \%$ ) among Swedish schoolchildren, and Scragg \& Shah found 1.5\% (95\% CI $0.6 \%$
$3.3 \%$ ) among men in Broadmoor. For figures including all possible cases, Ehlers \& Gillberg found a prevalence of $0.71 \%(95 \% \mathrm{CI} 0.34 \%-1.31 \%)$ and Scragg \& Shah found 2.3\% (95\% CI $1.1 \%-4.3 \%)$. It cannot therefore be reasonably concluded that the differences in prevalence found were not due to chance alone.

Given the recent publicity surrounding the case of the 13-year-old boy with AS who killed an 85-year-old woman in Wimborne, Dorset (Wright, 1994), it is important that readers do not conclude that AS is associated with a increased tendency to commit violent acts when this has not been proven.

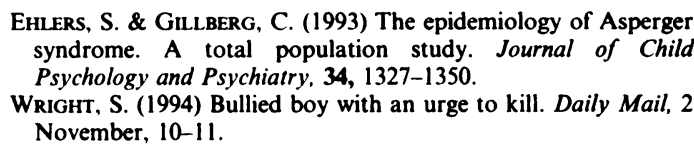

I. Hall

Division of Psychiatry of Disability

J. BERNAL

St. George's Hospital Medical School

London SW17 ORE

\section{Mental health reforms in Europe}

SIR: Grove's editorial on reform of mental health care in Europe (BJP, October 1994, 165, 431-433) was timely and wise. He correctly states that the medical role in psychiatry is increasingly being marginalised. This is very unfortunate as several of the developments in mental health care in recent years were initiated by psychiatrists.

Grove fails, however, to offer directions towards the future of mental health services either within the European Union or in Europe as a whole. The structural, organisational and funding changes in the health service systems occurring in most European countries will not leave the mental health services unaffected.

The current trends of staff skills mixing, the increasing participation of users and the quality of mental health service provision will be some of the issues to dominate the agenda in the next decade and beyond. What psychiatry will have to offer to 